\title{
ANÁLISE DA EFICIÊNCIA ENERGÉTICA DA BIBLIOTECA MUNICIPAL DE CAMPO MOURÃO-PR: PARÂMETROS DO PROGRAMA NACIONAL DE ETIQUETAGEM (RTQ-C)
}

\section{ARTIGO ORIGINAL}

CARVALHO, Fanny Islana de Lima1, HALMEMAN, Maria Cristina Rodrigues², LERCO, Felipe Matos dos Santos ${ }^{3}$

CARVALHO, Fanny Islana de Lima. HALMEMAN, Maria Cristina Rodrigues. LERCO, Felipe Matos dos Santos. Análise da eficiência energética da biblioteca municipal de Campo Mourão-PR: parâmetros do programa nacional de etiquetagem (RTQ-C). Revista Científica Multidisciplinar Núcleo do Conhecimento. Ano. 06, Ed. 10, Vol. 05, pp. 43-62. Outubro 2021. ISSN: 2448-0959, Link de acesso: https://www.nucleodoconhecimento.com.br/engenharia-ambiental/biblioteca-

municipal, $\quad$ DOI: 10.32749/nucleodoconhecimento.com.br/engenhariaambiental/biblioteca-municipal

\section{RESUMO}

O setor da construção civil, responsável por impactos ambientais como geração de resíduos, consumo de água e energia, tem como desafio atual trazer a eficiência energética para dentro do canteiro de obras e ambiente pós construído. Desse modo, em relação ao consumo de energia, o INMETRO desenvolveu um programa de etiquetagem visando classificar a eficiência energética de equipamentos elétricos e também edificações. Verifica-se que os edifícios públicos são obrigados a obter a Etiqueta Nacional de Conservação de Energia (ENCE). Diante do exposto, indagase como medir a eficiência energética de edifícios públicos, tornando-os adequados energeticamente, e assim atender as necessidades do usuário, proporcionando

\footnotetext{
${ }^{1}$ Bacharel em Arquitetura e Urbanismo. ORCID: 0000-0002-1871-2618.

2 Bacharel em Engenharia Civil. ORCID: 0000-0002-2809-6601.

${ }^{3}$ Doutorado em Ciências Agronômicas. ORCID: 0000-0001-6964-7572.
}

RC: 99090

Disponível em: https://www.nucleodoconhecimento.com.br/engenhariaambiental/biblioteca-municipal 
conforto ambiental e economia. O objetivo do trabalho foi simular a obtenção da etiqueta ENCE Geral e apresentar qual o nível de eficiência energética da Biblioteca Municipal de Campo Mourão-PR. Utilizou-se o Método Prescritivo, estabelecido pelo Regulamento Técnico da Qualidade para o Nível de Eficiência Energética de Edificações Comerciais, de Serviços e Públicas (RTQ-C). Foram analisados três sistemas da edificação: Envoltória, Sistema de Iluminação e Sistema de Condicionamento de Ar. Como resultado, o edifício em estudo apresentou classificação nível $C$ de eficiência, numa escala de $A$ - $E$, sendo $A$ muito eficiente, e $E$ pouco eficiente. Conclui-se que a edificação não proporciona conforto aos usuários, além de alto consumo de energia. Porém, é possível adotar medidas que podem auxiliar a otimização do nível de eficiência energética no local.

Palavras-chave: Envoltória, Sistema de lluminação, Condicionamento de Ar, Método Prescritivo, Classificação Total.

\section{INTRODUÇÃO}

A preocupação de países desenvolvidos com a conservação ambiental resultou em conferências internacionais, buscando alternativas econômicas. (DEBATES INTERDISCIPLINARES VII, 2016). Exemplos dessas conferências são Protocolo de Kyoto e a Convenção de Estocolmo. No cenário nacional, a partir de 2014 os prédios públicos passaram a ser obrigados a apresentar etiqueta de eficiência energética (ENCE), conforme publicado no Diário Oficial da União pela Secretaria de Logística e Tecnologia da normativa IN02/2014 (CONSELHO DE ARQUITETURA E URBANISMO/BR, 2014). Diante do exposto, surgiu a indagação sobre como é possível medir a eficiência energética de uma edificação pública, garantindo conforto e economia aos usuários.

O Programa Brasileiro de Etiquetagem (PBE), juntamente com o PBE Edifica surgiu a partir de discussões iniciadas pelo Instituto Nacional de Metrologia, Qualidade e Tecnologia (INMETRO). O objetivo deste programa é conscientizar o consumidor através de informações sobre o funcionamento de equipamentos e o seu consumo 
energético (CONFERÊNCIA DE ESTUDOS EM ENERGIA ELÉTRICA, 2019). Tal programa desenvolveu o Regulamento Técnico da Qualidade para o Nível de Eficiência Energética de Edificações Residenciais (RTQ-R) e os Requisitos Técnicos da Qualidade para o Nível de Eficiência Energética de Edifícios Comerciais, de Serviços e Públicos (RTQ-C). O sistema de etiquetagem mede o nível de eficiência energética em uma escala de " $A-E$ ", sendo " $A$ " mais eficiente, e "E" menos eficiente. O RTQ-C avalia edificações através de dois métodos: Método de Simulação (realizado por softwares) e Método Prescritivo (feito através de equações).

Há quatro tipos de etiquetas, sendo elas: ENCE Geral (leva em consideração três sistemas da edificação - envoltória, iluminação e condicionamento de ar); ENCE Parcial (avalia de forma independente cada sistema); ENCE Simulação (obtida ainda durante a fase de projeto); e ENCE Edificação Construída (podendo ser solicitada após a obtenção do alvará da obra ou comprovada ligações de energia elétrica e gás pelas concessionárias).

Este artigo objetivou identificar o nível de Eficiência Energética da Biblioteca Municipal simulando a obtenção da ENCE Geral. A análise foi desenvolvida em concordância com o Manual para Aplicação do RTQ-C versão 4, de 2017. Para atingir o objetivo geral, o presente trabalho se desenvolveu a partir dos seguintes objetivos específicos: Observar os pré-requisitos exigidos pelo Manual para Aplicação do RTQ-C; levantar dados in loco e processar informações do projeto arquitetônico; calcular e determinar o nível de eficiência energética para os três sistemas da edificação.

\section{MATERIAIS E MÉTODOS}

\subsection{MATERIAIS}

A atual Biblioteca Municipal Professor Egydio Martello localiza-se na área central de Campo Mourão-PR. A cidade possui aproximadamente 96.102 habitantes (IBGE, 
2020). Campo Mourão é polo da COMCAM (Comunidade dos Municípios da Região de Campo Mourão), a qual representa vinte e cinco municípios, desta maneira, a cidade oferece suporte para a sua microrregião por meio de assistência médica, educação, indústria entre outras áreas.

Figura 01. Localização da Biblioteca Municipal.

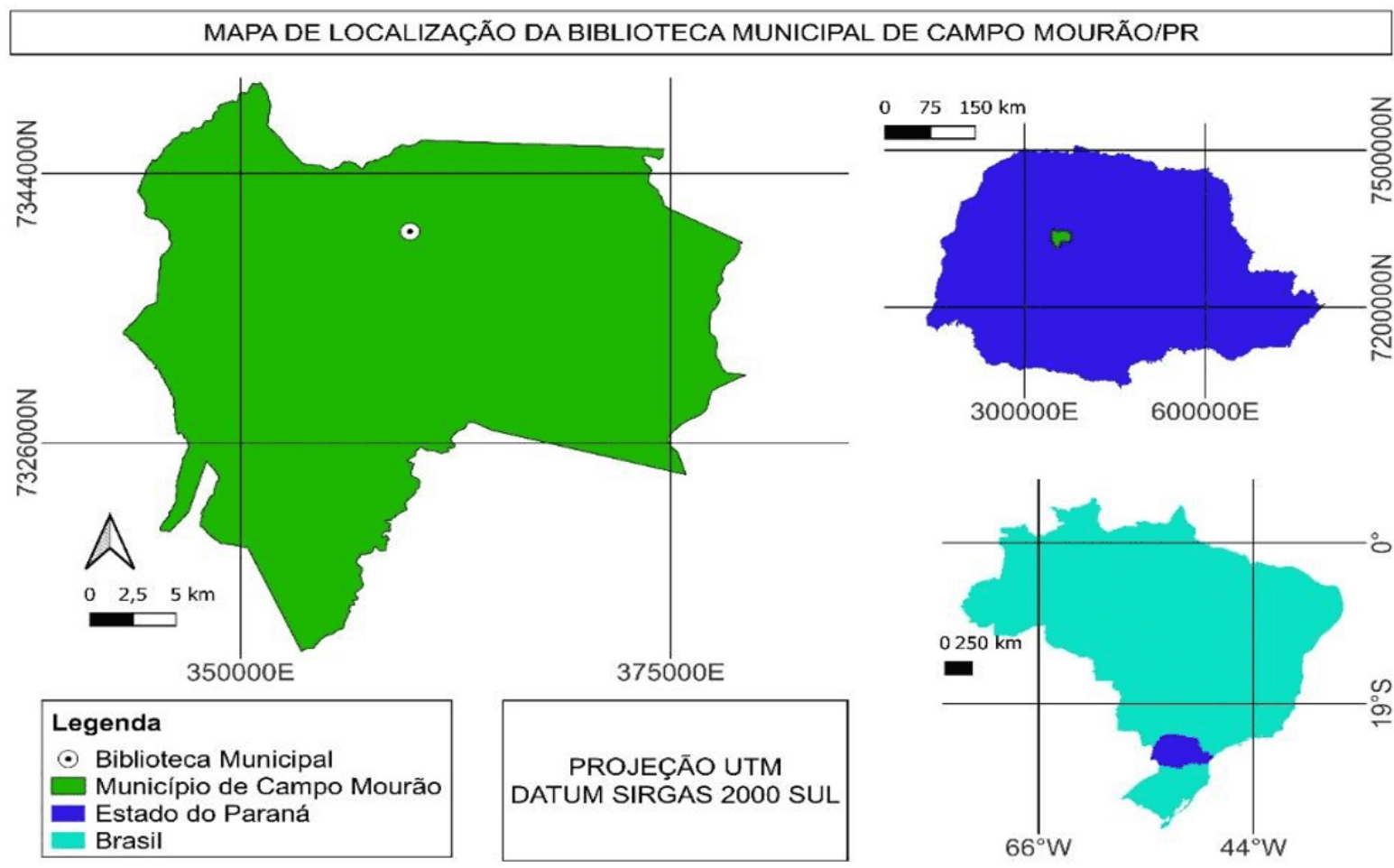

Fonte: Jairo Jerônimo de Campos Filho (2021).

O prédio em estudo apresenta grande relevância histórica no contexto do desenvolvimento do município, uma vez que este originalmente foi projetado para ser o Terminal Rodoviário. Posteriormente, a edificação foi adaptada para abrigar a biblioteca, a qual inaugurou em 30 de dezembro de 2004.

RC: 99090

Disponível em: https://www.nucleodoconhecimento.com.br/engenhariaambiental/biblioteca-municipal 
Figura 02. Antiga Rodoviária de Campo Mourão (A), atual Biblioteca Municipal (B), Fachada Principal Biblioteca Municipal (C).

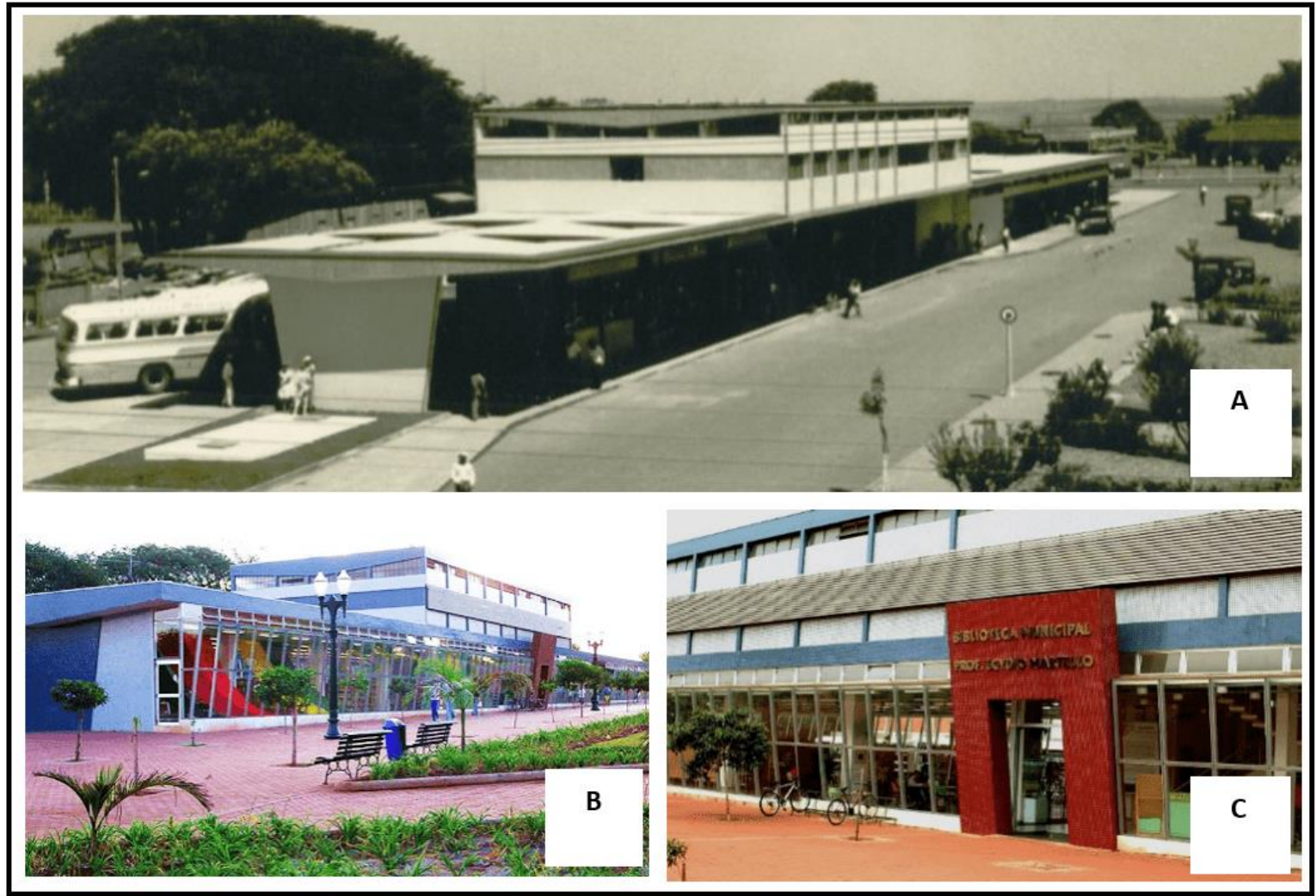

Fonte: (A) Acervo pessoal de Jair Elias dos Santos Júnior (1969) e (B, C) acervo da Biblioteca Municipal Professor Egydio Martello (2007).

Além de um vasto acervo, espaço de exposições artísticas, ludoteca, e áreas de estudo, a biblioteca também abriga a Academia Mourãoense de Letras. Trata-se de uma edificação linear, com dois pavimentos, em estrutura de concreto armado, com uma área total construída de $1772.92 \mathrm{~m}^{2}$, conforme o layout da Biblioteca na Figura 03. Grande parte da sua envoltória é composta por cortinas de vidro translúcido, o que favorece o aquecimento indesejado dos espaços internos. É importante ressaltar que sua fachada principal está voltada à direção nordeste, portanto, recebe alta incidência de radiação solar no período do verão.

RC: 99090

Disponível em: https://www.nucleodoconhecimento.com.br/engenhariaambiental/biblioteca-municipal 
Figura 03. Layout Biblioteca Pavimento Térreo (A), Escritórios Pavimento Térreo (B), Biblioteca Pavimento Superior (C).

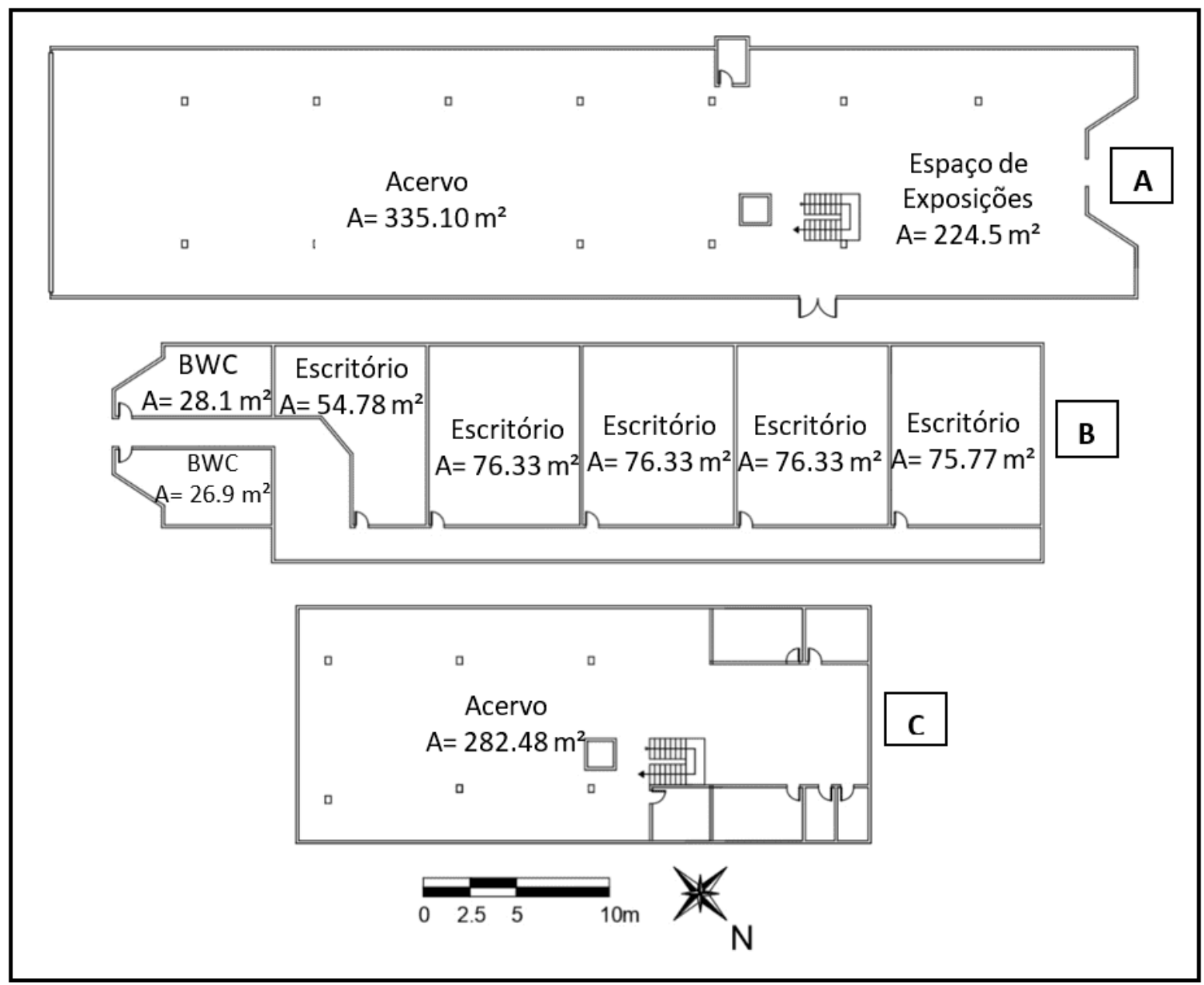

Fonte: Adaptado do Projeto Arquitetônico original (2003).

\subsection{MÉTODOS}

O método adotado para o presente estudo é o método prescritivo, o qual se aplica somente para edifícios condicionados e avalia a eficiência energética por meio de equações, de acordo com a Zona Bioclimática e a área total da edificação. Com o intuito de obter pontuação para a etiqueta da categoria ENCE Geral, foram analisados três sistemas: Envoltória, Sistema de Iluminação e Condicionamento de

RC: 99090

Disponível em: https://www.nucleodoconhecimento.com.br/engenhariaambiental/biblioteca-municipal 
Ar. Tal metodologia exige avaliação de pré-requisitos gerais e específicos de cada sistema, os quais serão discutidos a seguir.

\subsubsection{PRÉ-REQUISITOS GERAIS}

Para os níveis $\mathrm{A}, \mathrm{B}$ e $\mathrm{C}$, existem dois pré-requisitos gerais. $\mathrm{O}$ não cumprimento dos mesmos impactam na pontuação final da edificação.

- A edificação deve possuir um circuito elétrico distinto para cada uso, seja ele equipamentos de ar condicionado, lâmpadas ou outros. Porém, o Manual para Aplicação do RTQ-C (2017) estabelece que este item não se faz obrigatório para edificações construídas antes de 2009, portanto, o prédio em estudo está isento do mesmo.

- Aquecimento de Água: Este pré-requisito se aplica apenas em edifícios comerciais ou de serviço, onde o consumo de água quente é maior que 10\% da demanda total de energia. No caso do objeto de estudo, não há instalação de aquecedores de água, portanto, este item não se aplica.

\subsubsection{EQUAÇÕES PARA PROCEDIMENTO DE CÁLCULO}

No processo de classificação individual dos sistemas, assim como para a classificação geral da eficiência foram utilizadas as equações de acordo com o Quadro 01. 
Quadro 01. Quadro Geral de Equações.

\begin{tabular}{|c|c|c|}
\hline NOME & EQUAÇÃO & VARIÁVEIS \\
\hline $\begin{array}{c}\text { Equação } \\
01\end{array}$ & $\begin{array}{c}\text { IC env }=-14,14 . F A- \\
113,94 . F F+50,82 . P A F t o t a l+4,86 . F S- \\
\text { 0,32.AVS }+0,26 . A H S-\frac{35,75}{F F}- \\
\text { 0,54.PAFtotal.AHS }+277,98\end{array}$ & $\begin{array}{l}\text { IC env: Indicador de Consumo da } \\
\text { Envoltória. } \\
\text { Ape: Área de projeção do edifício } \\
\left(\mathrm{m}^{2}\right) ; \\
\text { Ato }_{\mathrm{t}} \text { : Área total construída }\left(\mathrm{m}^{2}\right) ; \\
\text { Aenv: Área da envoltória }\left(\mathrm{m}^{2}\right) ; \\
\text { Apcob: Área de projeção da } \\
\text { cobertura }\left(\mathrm{m}^{2}\right) ; \\
\text { AVS: Ângulo Vertical de } \\
\text { Sombreamento } \\
\text { AHS: Ângulo Horizontal de } \\
\text { Sombreamento } \\
\text { FF: Fator de Forma, (Aenv/ Vtot); } \\
\text { FA: Fator Altura, (Apcob/ Atot); } \\
\text { FS: Fator Solar; } \\
\text { PAF : Percentual de Abertura na } \\
\text { Fachada total: média do percentual } \\
\text { de aberturas existentes; }\end{array}$ \\
\hline
\end{tabular}

RC: 99090

Disponível em: https://www.nucleodoconhecimento.com.br/engenhariaambiental/biblioteca-municipal 


\begin{tabular}{|c|c|c|}
\hline $\begin{array}{c}\text { Equação } \\
05\end{array}$ & $\begin{array}{l}\mathbf{P T}=0,3 \cdot\left\{\left(\text { EqNumEnv } \frac{A C}{A U}\right)+\right. \\
\left.\left(\frac{A P T}{A U} \cdot 5+\frac{A N C}{A U} \cdot \text { EqNu } \mathrm{mV}\right)\right\}+0,3 . \\
(\text { EqNumDPl })+0,4 \cdot\{(\text { EqNumCA } . \\
\left.\left.\frac{A C}{A U}\right)+\left(\frac{A P T}{A U} \cdot 5+\frac{A N C}{A U} \cdot \text { EqNumV }\right)\right\}+ \\
\mathrm{b}_{0}^{1}\end{array}$ & $\begin{array}{l}\text { EqNumEnv: Equivalente Numérico } \\
\text { Envoltória } \\
\text { AC: Área Condicionada } \\
\text { AU: Área útil } \\
\text { APT: Área de permanência } \\
\text { Transitória } \\
\text { ANC: Área não condicionada } \\
\text { EqNumV: Equivalente Numérico } \\
\text { Área Ventilada } \\
\text { EqNumDPI: Equivalente Numérico } \\
\text { Densidade de Potência Instalada } \\
\text { EqNumCa: Equivalente Numérico } \\
\text { Condicionamento de Ar } \\
\text { b: Bonificações }\end{array}$ \\
\hline & & $\begin{array}{l}\text { Vto }: \text { Volume total da edificação } \\
\left(\mathrm{m}^{3}\right) \text {; }\end{array}$ \\
\hline $\begin{array}{c}\text { Equação } \\
02\end{array}$ & $\mathbf{i}=\frac{I C \text { máx }-I C \text { mín }}{4}$ & $\begin{array}{l}\text { i: Intervalo } \\
\text { IC } \mathbf{C}_{\max } \text { Indicador de Consumo } \\
\text { máximo } \\
\text { IC } \mathbf{C}_{\min }: \text { Indicador de Consumo } \\
\text { Mínimo }\end{array}$ \\
\hline $\begin{array}{c}\text { Equação } \\
03\end{array}$ & $\mathbf{P}_{\text {lim }}=\mathrm{A}^{2}$. DPIL & $\begin{array}{l}\text { PI }_{\text {im: Potência Limite }} \\
\text { A: Área iluminada } \\
\text { DPIL: Densidade de Potência } \\
\text { Instalada Limite }\end{array}$ \\
\hline $\begin{array}{c}\text { Equação } \\
04\end{array}$ & EqNumCA $=\sum_{n=1}^{x}:\left[\begin{array}{l}\text { EqNumCA } \\
n\end{array} \frac{\text { Capn }}{\text { Capt }}\right]$ & $\begin{array}{l}\text { EqNumCA: Equivalente Numérico } \\
\text { de Condicionamento de } \mathrm{Ar} \\
\text { Capn: Capacidade de cada } \\
\text { sistema } \\
\text { Capt: Capacidade total dos } \\
\text { sistemas } \\
\text { x: Quantidade de sistemas }\end{array}$ \\
\hline
\end{tabular}

Fonte: Adaptado do Manual para Aplicação para RTQ-C (2017).

\subsubsection{ENVOLTÓRIA}

Para atendimento dos pré-requisitos específicos do sistema da envoltória deve-se observar os valores das variáveis descritas no Quadro 02.

RC: 99090

Disponível em: https://www.nucleodoconhecimento.com.br/engenhariaambiental/biblioteca-municipal 
Quadro 02. Pré-requisitos Específicos para Envoltória - Zona 3.

\begin{tabular}{|c|c|c|c|c|c|c|}
\hline Nível & \multicolumn{3}{|c|}{ Transmitância Térmica (U) } & \multicolumn{2}{|c|}{$\begin{array}{c}\text { Cores e } \\
\text { Absortância } \\
\text { Térmica }(\alpha)\end{array}$} & $\begin{array}{c}\text { Iluminação } \\
\text { Zenital }\end{array}$ \\
\hline \multirow{2}{*}{ - } & \multicolumn{2}{|c|}{ Cobertura } & Parede & Cobertura & Parede & \multirow{6}{*}{ N/A } \\
\hline & $\begin{array}{c}\text { Ambiente } \\
\text { Condicionado }\end{array}$ & $\begin{array}{l}\text { Ambiente não } \\
\text { condicionado }\end{array}$ & - & \multicolumn{2}{|l|}{-} & \\
\hline A & $1.0 \mathrm{~W} / \mathrm{m}^{2} \mathrm{~K}$ & $2.0 \mathrm{~W} / \mathrm{m}^{2} \mathrm{~K}$ & $\begin{array}{c}3.7 \\
\mathrm{~W} / \mathrm{m}^{2} \mathrm{k} \\
\end{array}$ & $<0.50$ & $<0.50$ & \\
\hline B & $1.5 \mathrm{~W} / \mathrm{m}^{2} \mathrm{k}$ & $2.0 \mathrm{~W} / \mathrm{m}^{2} \mathrm{~K}$ & $\begin{array}{c}3.7 \\
\mathrm{~W} / \mathrm{m}^{2} \mathrm{k}\end{array}$ & $<0.50$ & $\mathrm{~N} / \mathrm{A}$ & \\
\hline C & \multicolumn{2}{|c|}{$2.0 \mathrm{~W} / \mathrm{m}^{2} \mathrm{~K}$} & $\begin{array}{c}3.7 \\
\mathrm{~W} / \mathrm{m}^{2} \mathrm{k}\end{array}$ & \multirow{2}{*}{\multicolumn{2}{|c|}{$\mathrm{N} / \mathrm{A}$}} & \\
\hline E & \multicolumn{2}{|c|}{$>2.0 \mathrm{~W} / \mathrm{m}^{2} \mathrm{~K}$} & $\begin{array}{c}>3.7 \\
\mathrm{~W} / \mathrm{m}^{2} \mathrm{~K}\end{array}$ & & & \\
\hline
\end{tabular}

Fonte: Adaptado do Manual para Aplicação do RTQ-C (2017).

A ABNT NBR 15220-2 (2008) estabelece parâmetros para o desempenho térmico de edificações. Após verificar o atendimento dos valores acima em conformidade com a norma, é necessário observar a Zona Bioclimática na qual o objeto de estudo está inserido. De acordo com o Anexo 1 do Manual para Aplicação do RTQ-C (2017), a cidade de Campo Mourão está na Zona Bioclimática 3, conforme mostra a Figura 04. 
Figura 04. Zoneamento Bioclimático do Brasil.

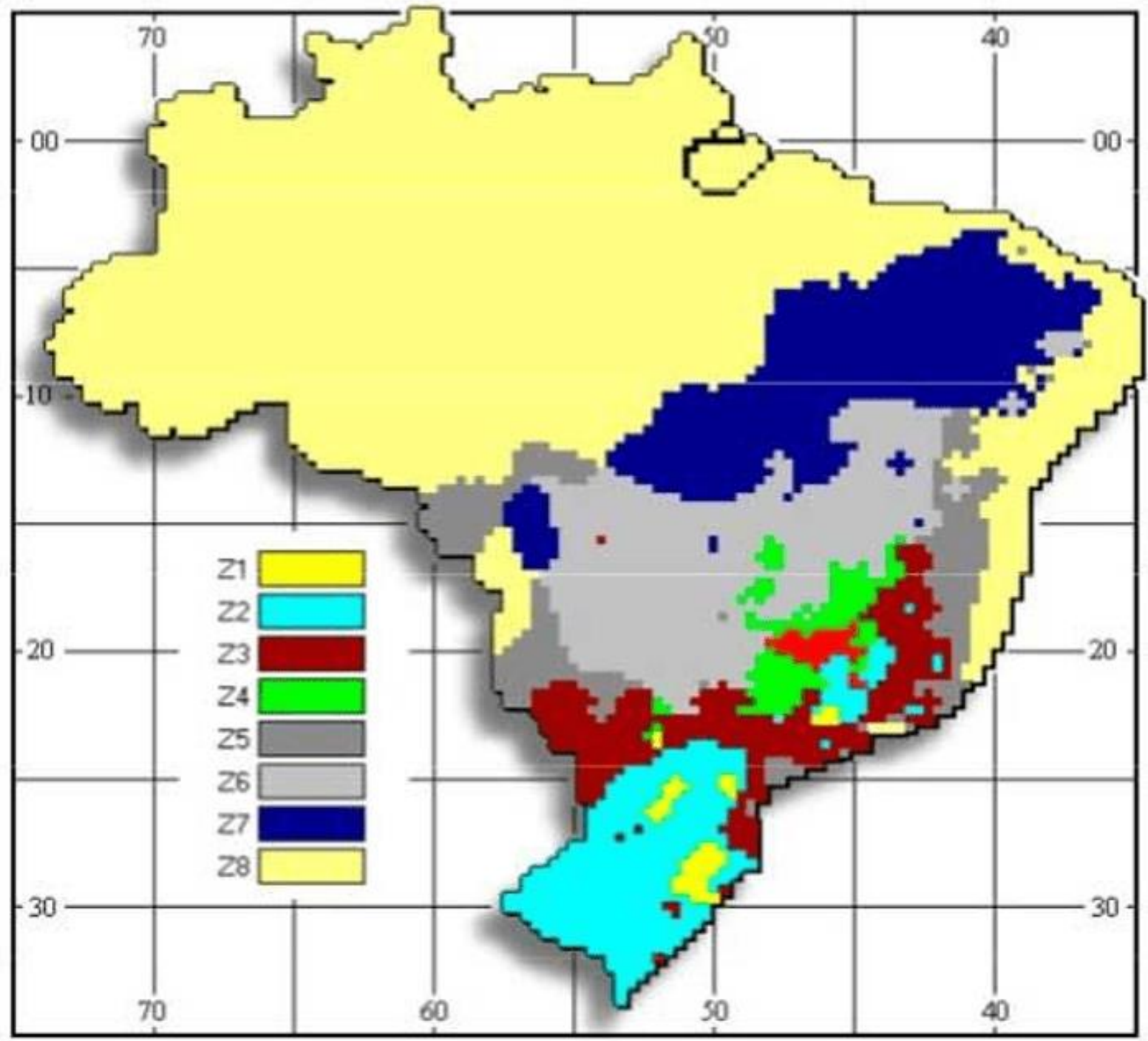

Fonte: Bioclimatismo (2021).

Para o procedimento de cálculo, foram necessárias as informações extraídas do projeto arquitetônico e as variáveis conforme o Quadro 01.

Considerando que o Manual especifica equações distintas de acordo com a área do objeto de estudo, para áreas maiores que $500 \mathrm{~m}^{2}$ a Equação 01 se enquadra na edificação.

RC: 99090

Disponível em: https://www.nucleodoconhecimento.com.br/engenhariaambiental/biblioteca-municipal 
Logo após, determinou-se o IC mínimo e IC máximo através da mesma equação, porém com os valores de entrada conforme o Quadro 03.

Quadro 03. Parâmetros do IC máximo e IC mínimo.

\begin{tabular}{|l|c|c|c|c|}
\hline & PAF & FS & AVS & AHS \\
\hline IC máximo & 0,60 & 0,61 & 0 & 0 \\
\hline IC mínimo & 0,05 & 0,87 & 0 & 0 \\
\hline
\end{tabular}

Fonte: Manual para aplicação do RTQ-C (2017).

Logo, o intervalo (i) de IC máximo e IC mínimo foi calculado pela Equação 02. Com o valor de (i), definiu-se o Quadro 04:

Quadro 04. Limites dos intervalos dos níveis de eficiência.

\begin{tabular}{|c|c|c|c|c|c|}
\hline Eficiência & A & B & C & D & E \\
\hline Lim. Mín. & N/A & $\begin{array}{c}\text { IC máx }- \\
3 i+0,01\end{array}$ & $\begin{array}{c}\text { IC máx }- \\
2 i+0,01\end{array}$ & $\begin{array}{c}\text { IC máx }-\mathrm{i} \\
+0,01\end{array}$ & $\begin{array}{c}\text { IC máx }+ \\
0,01\end{array}$ \\
\hline Lim. Máx. & $\begin{array}{c}\text { IC máx }- \\
3 \mathrm{i}\end{array}$ & IC máx $-2 \mathrm{i}$ & $\mathrm{IC}$ máx $-\mathrm{i}$ & $\mathrm{IC}$ máx & $\mathrm{N} / \mathrm{A}$ \\
\hline
\end{tabular}

Fonte: Manual para aplicação do RTQ-C (2017).

Por fim, a partir dos valores do Quadro 04, comparou-se o valor de IC envoltória com os limites IC máximo e IC mínimo para identificar o nível de eficiência.

\subsubsection{SISTEMA DE ILUMINAÇÃO}

O atendimento dos pré-requisitos específicos para o sistema de iluminação está descrito no Quadro 05. 
Quadro 05. Pré-requisitos para iluminação.

\begin{tabular}{|l|l|l|l|l|}
\hline Pré-Requisito & Nível A & Nível B & Nível C & Situação \\
\hline 1. Divisão dos circuitos & Exigido & Exigido & Exigido & Isento \\
\hline 2. Contribuição da luz natural & Exigido & Exigido & N/A & $\begin{array}{l}\text { Não } \\
\text { atende }\end{array}$ \\
\hline $\begin{array}{l}\text { 3. Desligamento automático } \\
\text { do sistema de iluminação }\end{array}$ & Exigido & N/A & N/A & $\begin{array}{l}\text { Não } \\
\text { atende }\end{array}$ \\
\hline
\end{tabular}

Fonte: Adaptado do Manual para Aplicação do RTQ-C (2017).

O prédio em estudo está isento do pré-requisito número 1, e não atende os demais pré-requisitos, desta forma, o nível do sistema de iluminação automaticamente apenas pode ter como classificação máxima o nível C. Porém, sua classificação final está subordinada aos cálculos de Densidade de Potência Instalada Limite.

Há dois métodos para o procedimento de cálculo da eficiência do sistema de iluminação:

- Método das Áreas: Avalia de forma conjunta os ambientes (para edificações com 3 atividades principais, ou atividades que ocupam mais de $30 \%$ da área edificada).

- Método das Atividades: Avalia de forma individual cada atividade.

Visto que a Biblioteca Municipal abriga 3 atividades principais, e que ocupam mais de $30 \%$ da área total, optou-se pelo Método das Áreas. O procedimento de cálculo se deu a partir das seguintes etapas:

a) Foram identificadas as atividades principais de acordo com a Tabela de Limite máximo aceitável de densidade de potência de iluminação (DPIL) para o nível de eficiência pretendido - Método da área da edificação, do Manual para Aplicação do RTQ-C (2017). Para atividades não descritas, optou-se por uma atividade equivalente. 
b) Foi identificada a área de cada atividade, em metros quadrados.

c) Em seguida, definiu-se a potência limite para cada atividade, multiplicando a área iluminada pela DPIL fornecida na mesma Tabela do Manual, conforme a Equação 03. A partir da potência limite para cada atividade, a soma das potências determinou a potência limite do edifício.

d) Por fim, comparou-se a potência total instalada no edifício com a potência limite para determinar o nível de eficiência do sistema de iluminação através do Equivalente Numérico fornecido no Quadro 06.

Quadro 06. Equivalente numérico (EqNum) para cada nível de eficiência.

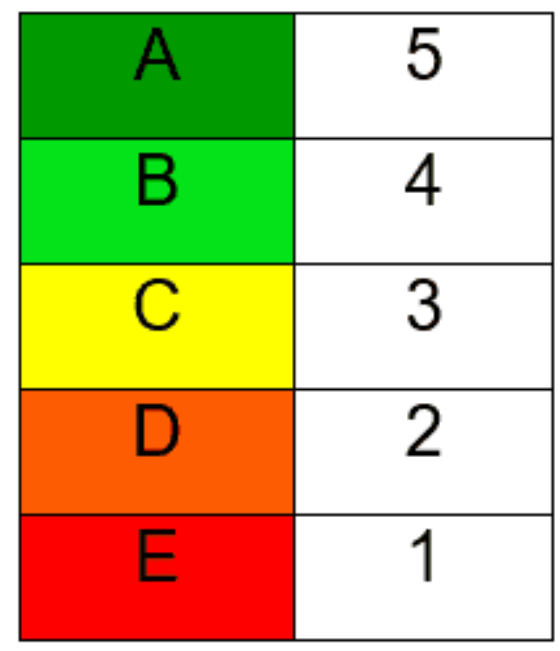

Fonte: Manual para Aplicação do RTQ-C (2017).

\subsubsection{CONDICIONAMENTO DE AR}

Os equipamentos de ar-condicionado instalados na Biblioteca Municipal são certificados pelo INMETRO, sendo assim, utilizou-se as informações técnicas fornecidas pelo próprio instituto e pelos fabricantes. O procedimento para determinação da eficiência do sistema foi segregado em etapas:

a) Observou-se a eficiência de cada sistema através da etiqueta no equipamento.

RC: 99090

Disponível em: https://www.nucleodoconhecimento.com.br/engenhariaambiental/biblioteca-municipal 
b) Determinou-se o Coeficiente de Ponderação por meio da razão entre a potência de cada unidade e a soma das potências de todas as unidades.

c) A partir do Coeficiente de Ponderação multiplicado pelo Equivalente Numérico conforme Quadro 06, obteve-se o Resultado Ponderado.

d) A somatória dos Resultados Ponderados de cada unidade determinou a eficiência total, conforme expressada na Equação 04.

Por existirem eficiências distintas, o sistema de Condicionamento de Ar deve ter seu Equivalente Numérico de acordo com o Quadro 07:

Quadro 07. Equivalente Numérico para Sistemas de Condicionamento de Ar e Pontuação Geral.

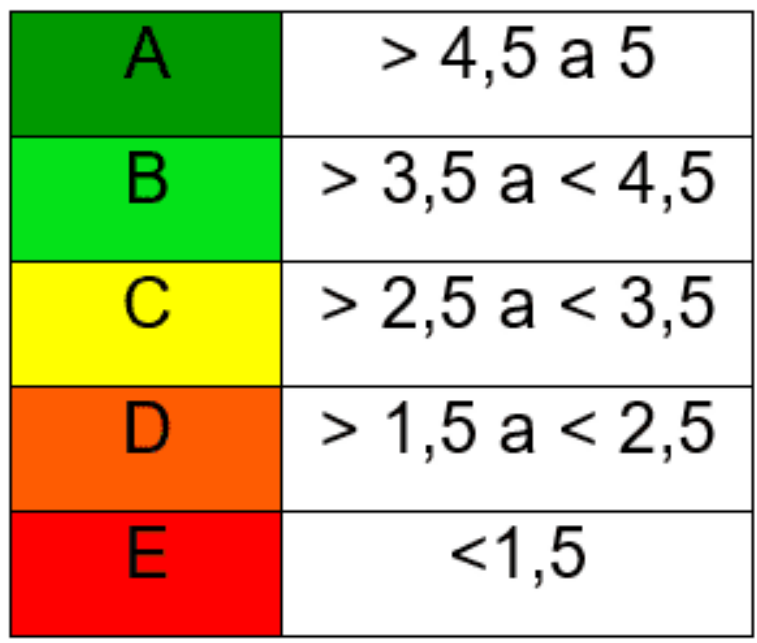

Fonte: Manual para Aplicação do RTQ-C (2017).

\subsubsection{PONTUAÇÃO TOTAL}

A determinação do nível de eficiência total do edifício é expressa através da Equação 05 , a qual atribui pesos para cada sistema: $30 \%$ para envoltória, 30\% para sistema de iluminação e $40 \%$ para sistema de condicionamento de ar. A partir do 
resultado da equação, é necessário converter o valor numérico determinado para cada nível, como mostra o Quadro 07.

\section{RESULTADOS E DISCUSSÃO}

\subsection{ENVOLTÓRIA}

Para analisar a envoltória, observou-se primeiramente, o cumprimento dos prérequisitos específicos para este sistema. O Quadro 08 demonstra a pontuação para cada pré-requisito.

Quadro 08. Atendimento dos Pré-requisitos Específicos.

\begin{tabular}{|c|c|c|c|c|}
\hline \multirow{4}{*}{ Nível } & \multicolumn{2}{|c|}{ Transmitância Térmica (U) } & \multicolumn{2}{|c|}{$\begin{array}{l}\text { Cores e Absortância } \\
\text { Térmica }(\alpha)\end{array}$} \\
\hline & Cobertura & Parede & Cobertura & Parede \\
\hline & $\begin{array}{c}\text { Telha } \\
\text { fibrociment } \\
00.7 \mathrm{~cm} \mathrm{U} \\
=1.75 \mathrm{Wm} \\
{ }^{2} \mathrm{~K}\end{array}$ & $\begin{array}{c}\text { Tijolo } 6 \text { furos } \\
\text { circulares, } \\
\text { assentados na } \\
\text { maior dimensão. U } \\
=1.88 \mathrm{Wm}^{-2} \mathrm{~K}\end{array}$ & $\begin{array}{c}\text { Telha } \\
\text { fibrociment } \\
\text { o } 0.7 \mathrm{~cm} \alpha \\
=0.30\end{array}$ & $\begin{array}{l}\text { Vidro Incolor } \\
\quad \alpha=0.25\end{array}$ \\
\hline & C & A & A & A \\
\hline
\end{tabular}

Fonte: Autoria própria.

Em seguida, determinou-se o Indicador de Consumo (IC) conforme a Equação 01.0 Quadro 09 indica os valores para cada variável, obtidos a partir do projeto arquitetônico.

RC: 99090

Disponível em: https://www.nucleodoconhecimento.com.br/engenhariaambiental/biblioteca-municipal 
Quadro 09. Variáveis de Projeto.

\begin{tabular}{|l|c|}
\hline \multicolumn{1}{|c|}{ Variáveis de Projeto } & Valores \\
\hline Ape: Área de projeção do edifício $\left(\mathrm{m}^{2}\right) ;$ & $799.97 \mathrm{~m}^{2}$ \\
\hline Atot: Área total construída $\left(\mathrm{m}^{2}\right) ;$ & $1599.95 \mathrm{~m}^{2}$ \\
\hline Aenv: Área da envoltória $\left(\mathrm{m}^{2}\right) ;$ & $2641.75 \mathrm{~m}^{2}$ \\
\hline Apcob: Área de projeção da cobertura $\left(\mathrm{m}^{2}\right) ;$ & $1280.13 \mathrm{~m}^{2}$ \\
\hline AVS: Ângulo Vertical de Sombreamento & $0^{*}$ \\
\hline AHS: Ângulo Horizontal de Sombreamento; & $0^{*}$ \\
\hline FF: Fator de Forma, (Aenv/ Vtot); & 0.401 \\
\hline FA: Fator Altura, (Apcob/ Atot); & 0.194 \\
\hline FS: Fator Solar; & 0.27 \\
\hline $\begin{array}{l}\text { PAFT: Percentual de Abertura na Fachada total: média do } \\
\text { percentual de aberturas existentes; }\end{array}$ & $34.76 \%$ \\
\hline Vtot: Volume total da edificação (m3 ); & $6575.15 \mathrm{~m}^{3}$ \\
\hline
\end{tabular}

Fonte: Autoria própria.

* Valores nulos, pois a edificação não apresenta beirais, tampouco elementos verticais de proteção solar.

Para determinar o Fator Solar, utilizou-se o vidro padrão comercial para edifícios Cool Lite 114 PN 8 mm, conforme especificado no Catálogo de Propriedades Térmicas e Óticas de Vidros Comercializados no Brasil. Após o cálculo, obteve-se como resultado o Indicador de Consumo da Envoltória (IC) igual a 159.373.

Em seguida, calculou-se o IC máximo ( $\left(\mathrm{C}_{\max }\right)$ e IC mínimo ( $\left(\mathrm{C}_{\min }\right)$, utilizando também a Equação 01, porém substituindo pelos valores descritos no Quadro 04. Como resultado, concluiu-se que IC $\max$ é igual a 173,852 e IC miné igual a 147.165.

Para calcular o Intervalo (i) $I C_{\max }$ e $I C_{\min }$ aplicou-se a Equação 02, onde o resultado obtido foi 6.671 .

A partir do resultado de (i), substituiu-se os valores de (i) do Quadro 05 por 6.671, resultando no Quadro 10, o qual apresenta os limites mínimos e máximos para cada nível de eficiência.

RC: 99090

Disponível em: https://www.nucleodoconhecimento.com.br/engenhariaambiental/biblioteca-municipal 
Quadro 10. Valores de ICenv máximo e mínimo para cada nível.

\begin{tabular}{|c|c|c|c|c|c|}
\hline \multirow{5}{*}{ Mínimo } & A & B & C & $\bar{D}$ & E \\
\hline & \multirow{4}{*}{$\mathrm{N} / \mathrm{A}$} & $\begin{array}{c}\text { IC máx }-3 i+ \\
0,01\end{array}$ & $\underset{0,01}{I C}$ máx $-2 i+$ & $\begin{array}{c}\text { IC máx - i + } \\
0,01\end{array}$ & IC máx $+0,01$ \\
\hline & & $\begin{array}{c}173,852-3 \\
(6,671)+ \\
0,01\end{array}$ & $\begin{array}{c}173,852-2 \\
(6,671)+ \\
0,01\end{array}$ & $\begin{array}{c}173,852- \\
6,671+0,01\end{array}$ & $173,852+0,01$ \\
\hline & & $\begin{array}{c}173,852- \\
20,013+ \\
0,01 \\
\end{array}$ & $\begin{array}{c}173,852- \\
13,342+ \\
0,01 \\
\end{array}$ & & \\
\hline & & *153,849 & 160,52 & 167,191 & 173,862 \\
\hline \multirow{5}{*}{ Máximo } & $\mathrm{A}$ & $B$ & C & D & E \\
\hline & IC máx - 3i & IC máx - 2i & IC máx - i & IC máx & \multirow{4}{*}{ N/A } \\
\hline & $\begin{array}{c}173,852-3 \\
(6,671)\end{array}$ & $\begin{array}{c}173,852-2 \\
(6,671)\end{array}$ & $\begin{array}{c}173,852- \\
6,671\end{array}$ & \multirow[b]{3}{*}{173,852} & \\
\hline & $\begin{array}{c}173,852- \\
20,013\end{array}$ & $\begin{array}{c}173,852- \\
13,342 \\
\end{array}$ & \multirow[b]{2}{*}{167.181} & & \\
\hline & 153,839 & *160,51 & & & \\
\hline
\end{tabular}

Fonte: Autoria própria.

* Valor real do IC env da Biblioteca com os limites encontrados, em que o nível de eficiência da Envoltória é B $(160,51<159,373$ > 153,849).

\subsection{SISTEMA DE ILUMINAÇÃO}

Para determinar o nível de eficiência do Sistema de lluminação utilizando o Método das Áreas, foram identificadas as atividades desenvolvidas em cada espaço da Biblioteca. $O$ atendimento dos pré-requisitos foi observado individualmente para cada ambiente, por fim, a análise do sistema como um todo foi realizada através da soma das DPIL de todos os ambientes. O procedimento de cálculo se deu a partir das informações contidas no Quadro 11.

RC: 99090

Disponível em: https://www.nucleodoconhecimento.com.br/engenhariaambiental/biblioteca-municipal 
Quadro 11. Classificação do Sistema de Iluminação.

\begin{tabular}{|c|c|c|c|c|c|}
\hline \multicolumn{2}{|l|}{ Atividade } & $\begin{array}{c}\text { Biblioteca } 1^{\circ} \\
\text { Pavimento }\end{array}$ & $\begin{array}{c}\text { Biblioteca } 2^{\circ} \\
\text { Pavimento }\end{array}$ & Escritório & $\begin{array}{c}\text { Total } \\
\text { Edifício* }\end{array}$ \\
\hline \multicolumn{2}{|l|}{ Área lluminada $\left(\mathrm{m}^{2}\right)$} & 559.6 & 282.48 & 359.63 & 1201.71 \\
\hline \multirow{4}{*}{ DPIL RTQ-C } & A & \multicolumn{2}{|c|}{12.7} & 9.7 & \multirow{4}{*}{$\mathrm{N} / \mathrm{A}$} \\
\hline & B & \multicolumn{2}{|c|}{14.6} & 11.2 & \\
\hline & C & \multicolumn{2}{|c|}{16.5} & 12.6 & \\
\hline & D & \multicolumn{2}{|c|}{18.4} & 14.1 & \\
\hline \multirow{2}{*}{ Lâmpadas } & $\begin{array}{l}40 \\
\text { W }\end{array}$ & 90 units & 104 units & 146 units & 340 \\
\hline & $\begin{array}{l}18 \\
W\end{array}$ & 142 units & 0 & 0 & 142 \\
\hline \multicolumn{2}{|c|}{ Potência Instalada (W) } & 6156 & 4160 & 5840 & 16156 \\
\hline \multirow{4}{*}{ Potência Limite (W) } & A & 7106.92 & 3587.49 & 3488.41 & 14182.82 \\
\hline & B & 8170.16 & 4124.2 & 4027.85 & 16322.21 \\
\hline & C & 9233.4 & 4660.92 & 4531.33 & 18425.65 \\
\hline & D & 10296.64 & 5197.63 & 5070.78 & 20565.05 \\
\hline \multicolumn{2}{|l|}{ Classificação Edifício } & \multicolumn{4}{|c|}{ Nível B } \\
\hline
\end{tabular}

Fonte: Autoria própria.

* A Área lluminada Total do Edifício em metros quadrados representa apenas as áreas de permanência prolongada, ou seja, não inclui as áreas secundárias como, banheiros, copa, circulação e outros. Uma vez que as equações descritas no Manual para Aplicação do RTQ-C já preveem uma margem considerando essas áreas secundárias.

Conforme os cálculos da DPIL de cada ambiente conforme a Equação 03, o Sistema de lluminação se classifica como nível B. Porém, considerando-se que o prédio em estudo não atende os pré-requisitos conforme o Quadro 05, a pontuação final do Sistema de lluminação cai para Nível C.

\subsection{CONDICIONAMENTO DE AR}

Para determinar a eficiência do sistema de Condicionamento de Ar, foram realizados os cálculos conforme a metodologia, e considerando que todos os equipamentos de

RC: 99090

Disponível em: https://www.nucleodoconhecimento.com.br/engenhariaambiental/biblioteca-municipal 
ar-condicionado da Biblioteca são do tipo Split Teto, obteve-se os seguintes resultados conforme demonstrado no Quadro 12.

Quadro 12. Classificação do Sistema de Condicionamento de Ar.

\begin{tabular}{|c|c|c|c|c|c|c|}
\hline Ambiente & $\begin{array}{c}\text { Biblioteca 10 } \\
\text { Pavimento }\end{array}$ & $\begin{array}{c}\text { Biblioteca 2 } \\
\text { Pavimento }\end{array}$ & \multicolumn{3}{|c|}{ Escritório } \\
\hline Fabricante & ELGIN & ELGIN & GREE & RHEEM & KOMECO & AUSTIN \\
\hline Quantidade & 3 & 2 & 2 & 2 & 1 & 1 \\
\hline $\begin{array}{c}\text { Potência } \\
\text { (Btu/h) }\end{array}$ & 60000 & 60000 & 9000 & 20000 & 57000 & 60000 \\
\hline $\begin{array}{c}\text { Classificação } \\
\text { Energética }\end{array}$ & $\mathrm{C}$ & $\mathrm{C}$ & $\mathrm{A}$ & $\mathrm{D}$ & $\mathrm{D}$ & $\mathrm{D}$ \\
\hline $\begin{array}{c}\text { Equivalente } \\
\text { Numérico }\end{array}$ & 3 & 3 & 5 & 2 & 2 & 2 \\
\hline $\begin{array}{c}\text { Coeficiente } \\
\text { de }\end{array}$ & 0.22 & 0.22 & 0.03 & 0.07 & 0.21 & 0.22 \\
\hline $\begin{array}{c}\text { Ponderação } \\
\text { Resultado } \\
\text { Ponderado }\end{array}$ & 0.66 & 0.66 & 0.15 & 0.14 & 0.42 & 0.66 \\
\hline
\end{tabular}

Fonte: Autoria própria. A partir da aplicação da Equação 04, o resultado obtido foi 2.69, o qual, de acordo com a Quadro 07, equivale ao nível C de eficiência.

\subsection{PONTUAÇÃO TOTAL (PT)}

Para definir o nível de eficiência energética geral do edifício, os resultados de cada sistema individual foram sintetizados na Equação 05. O Quadro 13 apresenta os valores para cada variável utilizada.

RC: 99090

Disponível em: https://www.nucleodoconhecimento.com.br/engenhariaambiental/biblioteca-municipal 
Quadro 13. Variáveis da Equação 05.

\begin{tabular}{|l|c|}
\hline EqNumEnv & 4 \\
\hline AC & $1201,71 \mathrm{~m}^{2}$ \\
\hline AU & $1592,73 \mathrm{~m}^{2}$ \\
\hline APT & $182,86 \mathrm{~m}^{2}$ \\
\hline ANC & $\mathrm{N} / \mathrm{A}^{*}$ \\
\hline EqNumV & $\mathrm{N} / \mathrm{A}^{*}$ \\
\hline EqNumDPI & 4 \\
\hline EqNumCA & 3 \\
\hline B & ${\mathrm{N} / \mathrm{A}^{*}}$ \\
\hline
\end{tabular}

Fonte: Autoria própria.

* Variáveis não se aplicam para ambientes condicionados.

O resultado de PT foi 3.111, enquadrando-se no nível $\mathrm{C}$ de eficiência energética.

\section{CONSIDERAÇÕES FINAIS}

Conclui-se que a Biblioteca Municipal de Campo Mourão não apresenta resultados satisfatórios de eficiência energética. Para otimizar sua eficiência, sugere-se:

- Intervenção na Envoltória com instalação de elementos de proteção solar, tais como beirais horizontais e verticais, brises, cobogós, entre outros.

- Em relação ao Sistema de Iluminação, recomenda-se a divisão dos circuitos, de maneira que possibilite o desligamento das lâmpadas próximas às janelas para o aproveitamento da luz natural.

- Quanto ao Sistema de Condicionamento de Ar, é importante substituir os atuais equipamentos que não possuem eficiência $A$, uma vez que $36 \%$ dos equipamentos apresentam classificação D. 
- Além dessas medidas, também se recomenda a substituição das telhas de fibrocimento por telhas termoacústicas.

- Para tornar a edificação mais sustentável, aconselha-se a instalação de placas fotovoltaicas para geração de energia solar, uma vez que o investimento inicial é compensado em curto prazo, proporcionando economia, baixa manutenção e funcionalidade.

\section{REFERÊNCIAS}

ASSOCIAÇÃO BRASILEIRA DE NORMAS TÉCNICAS. NBR 15220-2: Desempenho térmico de edificações: Métodos de cálculo da transmitância térmica, da capacidade térmica, do atraso térmico e do fator solar de elementos e componentes de edificações. 2008.

CONSELHO DE ARQUIETURA E URBANISMO DO BRASIL (Brasil). Governo torna obrigatório que edifícios públicos federais tenham energia eficientes. Disponível em: https://www.caubr.gov.br/governo-torna-obrigatorio-que-edificiospublicos-federais-sejam-energeticamente-eficientes/\#comments. Acesso em: 28 ago. 2021.

CONFERÊNCIA DE ESTUdOS EM ENERGIA ELÉTRICA. PROGRAMA BRASILEIRO DE ETIQUETAGEM: Breve Panorama. Uberlândia, MG: Universidade Federal de Uberlândia, 2019. 5 p. Disponível em: https://www.lunasat.top/ceel/evento/artigos/\#2019. Acesso em: 28 ago. 2021.

CENTRO BRASILEIRO DE EFICIÊNCIA ENERGÉTICA EM EDIFICAÇÕES. Catálogo de Propriedades Térmicas e Óticas de Vidros Comercializados No Brasil. Brasil: Universidade Federal de Santa Catarina, 2015. Disponível em: https://cb3e.ufsc.br/sites/default/files/projetos/etiquetagem/catalogopropriedades-vidros-comercializados-brasil-13032015_v2.pdf. Acesso em: 28 jul. 2021. 
DOS SANTOS JUNIOR, J.E. Antiga Rodoviária de Campo Mourão-PR. 1969. Fotografia.

FILHO, Jairo Jerônimo De Campos. Mapa Localização Biblioteca. Campo Mourão: 2021. Mapa em escala gráfica. Software QGIS V. 3.2.

Programa Nacional De Conservação De Energia Elétrica. Manual para aplicação do RTQ-C. 4 ed. Brasil, 2017.

HARADA, Magali. Bioclimatismo: Blog de Arquitetura Bioclimática. Disponível em: http://bioclimatismo.com.br/bioclimatismo/zoneamento-bioclimatico-brasileiro/. Acesso em: 05 jun. 2021.

IBGE - INSTITUTO BRASILEIRO DE GEOGRAFIA E ESTATÍSTICA. Censo Brasileiro de 2020. Rio de Janeiro. 2021.

DEBATES INTERDISCIPLINARES VII. O Papel das Conferências Internacionais Sobre o Meio Ambiente Para o Desenvolvimento dos Regimes Internacionais Ambientais: De Estocolmo A Rio +20. Palhoça, Sc: Unisul, 2016. Disponível em: https://www.researchgate.net/profile/Jose-Baltazar-Andrade-

Guerra/publication/301626018_Debates_Interdisciplinares_VII/links/571e496f08aeac ed7889df5e/Debates-Interdisciplinares-VII.pdf\#page=168. Acesso em: 20 ago. 2021.

Enviado: Agosto, 2021.

Aprovado: Outubro, 2021.

RC: 99090

Disponível em: https://www.nucleodoconhecimento.com.br/engenhariaambiental/biblioteca-municipal 